Revista de

Contabilidade e

Organizações

www.rco.usp.br
DOI: http://dx.doi.org/10.11606/issn.1982-6486.rco.2019.160869

Journal of

Accounting and

Organizations

www.rco.usp.br

\title{
Previsão de retornos e preços das ações a partir de dados contábeis condicionada ao ciclo de vida das firmas
}

Returns and stock prices forecast from accounting data conditioned on the firm life cycle

Karina da Silva Carvalho Mikosz ${ }^{a}$; Carolina Magda da Silva Roma ${ }^{\mathrm{b}}$; Luiz Cláudio Louzada ${ }^{\mathrm{c}}$; Marcos Roberto Gois de Oliveira Macedo ${ }^{\mathrm{a}}$

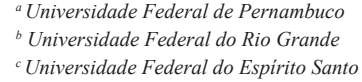

Palavras-chave

Modelo contábil.

Retorno e preço esperados.

Ciclo de vida da firma.

Estágios do ciclo de vida da firma.

\section{Resumo}

Este artigo investiga o efeito dos estágios do ciclo de vida das firmas na determinação dos retornos e preços esperados de ações brasileiras a partir do modelo contábil de avaliação desenvolvido por Lyle, Callen e Elliott (2013). Para definição dos estágios do ciclo de vida, adotou-se a metodologia de Dickinson (2011), utilizando uma proxy parcimoniosa para as firmas, estruturada a partir da demonstração do fluxo de caixa com forte aderência as teorias econômicas. Os dados foram coletados nas bases de dados Bloomberg, Quantum e do NEFIN/FEA/USP e as informações foram geradas no nível das firmas. Os resultados sugerem que o modelo para o período analisado não apresenta capacidade de previsão de retornos no Brasil, dada pela não significância das variáveis mesmo considerando os ativos em diferentes estágios de ciclo de vida da firma, já para as previsões de preços houve considerável poder explicativo, especialmente no estágio de maturidade.

\begin{abstract}
This article investigates the effect of firm's life cycle stages on the determination of expected returns and prices of Brazilian stocks based on the valuation accounting model developed by Lyle, Callen and Elliott (2013). In order to define the life cycle stages, we adopted the methodology developed by Dickinson (2011), using a parsimonious proxy for firm level, structured from the cash flow demonstration with strong economic theories adherence. Data were collected in the Bloomberg, Quantum and NEFIN/FEA/USP databases, the information was generated on the firm's level. The results suggest that the model for the analyzed period does not have returns predictions capacity in Brazil, given the non-significance of the variables even considering the assets in different stages of the firm life cycle, however for the prices' forecasts there was considerable explanatory power, especially in the maturity stage.
\end{abstract}

\section{Implicações práticas}

Os resultados sugerem que ao considerar os estágios do ciclo de vida das firmas é possível melhorar a previsão do preço das ações para as empresas brasileiras de capital aberto. Além disso, destaca-se que os dividendos são relevantes para a previsão dos preços nos diferentes ciclos de vida das firmas. Tais resultados podem auxiliar os investidores em suas previsões para o mercado brasileiro.

Copyright (C) 2019 FEA-RP/USP. Todos os direitos reservados
Recebido: 08 de agosto de 2019

Aceito: 05 de novembro de 2019

Publicado: 21 de novembro de 2019

E-mail: karina.carvalhoadm@gmail.com (K. da S. C. Mikosz); carolina.magda.adm@gmail.com (C. M. da S. Roma); louzadalvi@yahoo.com.br (L. C. Louzada); 


\section{INTRODUÇÃO}

A teoria do ciclo de vida da firma é um tema que vem ganhando visibilidade nos estudos de contabilidade e finanças. De acordo com esta teoria, as firmas passam por diversos padrões previsíveis de desenvolvimento, onde recursos, capacidades, estratégias, estruturas e funcionamento se modificam expressivamente em decorrência de seus estágios de desenvolvimento (Hasan, Hossain, Cheung, \& Habib, 2015). Dessa forma, a compreensão dos ciclos de vida pode contribuir para as firmas utilizarem e combinarem recursos de forma mais eficiente.

Os ciclos de vida das firmas são estágios distintos resultantes de mudanças em fatores internos como escolha de estratégia, recursos financeiros e capacidade gerencial, que ocorrem em função das atividades estratégicas realizadas e possuem implicações para compreensão do desempenho financeiro das firmas (Dickinson, 2011).

Dickinson (2011) desenvolveu uma proxy parcimoniosa no que concerne as firmas, para identificação dos estágios de ciclo de vida, estruturada a partir das práticas previstas dos fluxos de caixa operacionais, de investimento e de financiamento, obtendo uma metodologia de classificação resultante das escolhas de origem e alocação de recursos.

Desde então sua metodologia passou a ser adotada, sendo que alguns trabalhos merecem destaque, como Hasan et al. (2015) por mostrar que o custo do capital próprio varia ao longo do ciclo de vida da firma, para empresas australianas no período de 1990 e 2012. E ainda, Hasan e Cheung (2018) que abordam a associação entre o capital organizacional e o ciclo de vida das firmas, sugerindo que as firmas com alto (baixo) capital organizacional tem maior propensão de estarem nos estágios de introdução e declínio (crescimento e maturidade). Os autores descobriram também que as firmas com maiores investimentos em capital organizacional são menos (mais) tendentes, para um horizonte futuro de cinco anos, passarem para os estágios de introdução, shake-out e declínio (crescimento e maturidade).

Recentemente, Dickinson, Kassa e Schaberl (2018) examinaram o papel das informações contábeis em relação às previsões de lucros dos analistas na determinação de quais informações são mais relevantes para explicar o valor de mercado condicionado ao estágio do ciclo de vida da firma. Tanto em relação ao retorno, quanto ao preço foi observado que: i) nos estágios de crescimento e maturidade, os investidores atribuem peso relativamente maior às previsões dos analistas e; ii) nos estágios de introdução e declínio, os investidores consideram as informações contábeis mais relevantes.

Nesse contexto esta pesquisa objetiva verificar a capacidade do modelo de avaliação contábil recentemente desenvolvido por Lyle et al. (2013) na determinação dos retornos e preços esperados de ações brasileiras levando em consideração o efeito dos diferentes estágios do ciclo de vida das firmas. Este modelo amplia a estrutura de avaliação contábil de Ohlson (1995) e Feltham e Ohlson (1995, 1999), incorporando as expectativas dinâmicas sobre o nível de risco sistemático na economia em sua metodologia. Além disso, mostra uma forte associação negativa entre as alterações no risco de toda a economia e retornos futuros de ações e gera uma formulação explícita para o custo de capital. Especificamente, os autores definiram que o custo de capital é uma função linear de variáveis contábeis e outros fundamentos, que são o índice book-to-market, a relação do preço-lucro e preçolucro futuro, o tamanho e o dividend yield, sendo, portanto, de fácil implementação. Os resultados dos autores mostraram que o modelo consegue capturar a dinâmica dos retornos esperados e preços usando uma amostra de firmas listadas no mercado de capitais americano.

Contudo, uma vez que, as evidências presentes nos estudos de Hasan et al. (2015) e Dickinson et al. (2018) identificaram que o ciclo de vida influencia no custo de capital e previsão de preços e retornos a partir do modelo de Ohlson, respectivamente, aqui procurou-se investigar a ideia de que o mesmo influencia os resultados das regressões de retorno e de preço no modelo desenvolvido por Lyle et al. (2013).

$\mathrm{O}$ estudo desenvolvido se mostra relevante ao analisar as previsões de retorno e preço, em um ambiente de informações menos precisas, como é caso do Brasil, que torna a coleta, a produção e a análise de informações mais onerosas. Ademais, conforme apontando acima, a variável previsão de lucros por ação pelos analistas de mercado é integrante do modelo sob análise. Diante disso, Saito, Villalobos e Benettui (2008) afirmam que o Brasil é caracterizado por maiores incertezas sobre os lucros futuros e o valor intrínseco das ações, o que pode ocasionar: erros de previsão, variações entre as previsões dos analistas para a mesma firma e maior volatilidade dos retornos das ações. Nesse estudo encontraram uma tendência de viés positivo nas previsões dos analistas de mercado. Gatsios e Lima (2014) também demostram a existência de um viés positivo nas previsões dos analistas, no Brasil, de 2006 a 2012. Os autores apontam uma redução do viés nas previsões dos analistas no período de adoção das International Financial Reporting Standards (IFRS), e um aumento do viés durante o período de adoção parcial. 
Já Antônio, Lima e Pimenta Junior (2015) mostraram que os analistas brasileiros no período de 2000 a 2010 não conseguiram aconselhar os investidores acerca de ações lucrativas. Talvez porque como sugerido por Antônio, Ambrozini, Gatsios e Magnani (2017) o Brasil possui um número elevado de analistas de mercado, mas com pouco consenso entre eles quando comparado as médias dos países da América Latina abordados no estudo. Assim, como a previsão dos lucros pelos analistas é usado no modelo de Lyle et al. (2013), o contexto brasileiro fornece um ambiente propício para o estudo.

Para fins da pesquisa, foram estimadas regressões cross-section de Fama e MacBeth (1973) usando dados entre os anos de 2010 a 2016 para estimar o modelo de Lyle et al. (2013) e condicionando ao ciclo de vida com base na definição de Dickinson (2011). Os resultados evidenciaram que o modelo analisado não demonstrou significância estatística para previsões de retorno no mercado brasileiro no período em análise, mesmo quando os ativos foram considerados em diferentes estágios de ciclo de vida da firma, entretanto para as previsões de preços os resultados foram robustos, especialmente no estágio de maturidade.

\section{REFERENCIAL TEÓRICO}

Formado por uma fundamentação em várias áreas de pesquisa, como comportamento de produção, aprendizagem/experiência, investimento, participação de mercado e padrões de entrada/saída, Dickinson (2011) fornece uma medida de ciclo de vida específica da firma usando dados da demonstração de fluxo de caixa para identificar os estágios do ciclo de vida da firma (Habib \& Hasan, 2019).

Dickinson (2011) argumenta que os fluxos de caixa capturam diferenças na lucratividade, crescimento e risco de uma firma e, portanto, pode-se tomar os resultados dos fluxos de operação, de investimento, e de financiamento para agrupar as firmas em diferentes estágios do ciclo de vida, sendo eles: introdução, crescimento, maturidade, shake-out e declínio. Portanto, desenvolveu proxies para os estágios do ciclo de vida firma, que são derivadas da teoria econômica. Segundo Dickinson (2011), diversificando os sinais positivos e negativos das atividades principais dos fluxos de caixa líquidos é possível formar oito padrões de fluxo de caixa, posteriormente agregados em cinco estágios do ciclo de vida da firma, fornecendo assim um mapeamento em um determinado período da demonstração financeira.

Os principais resultados de Dickinson (2011) comprovaram que a proxy do padrão de fluxo de caixa é superior a outras proxies do ciclo de vida existentes na literatura (incluindo a idade da firma). Como também, explica melhor a rentabilidade futura tanto nas taxas de retorno, quanto nos retornos dos ativos. Trabalhos como Hasan et al. (2015), Koh, Durand, Dai e Chang (2015) e Faff, Kwok, Podolski e Wong (2016) utilizaram a teoria do ciclo de vida da firma para estudos nas áreas de contabilidade e finanças.

O estágio do ciclo de vida da firma, identificado pelos padrões de fluxo de caixa, deve ser uma perspectiva pela qual os investidores ou outros agentes avaliam o valor de mercado (Dickinson, 2011). Entretanto, o mercado não incorpora completamente as informações do ciclo de vida no preço dos ativos (Dickinson, 2011; Vorst \& Yohn, 2018), por isso firmas maduras alcançam excessos de retornos positivos no ano subsequente ao enquadramento da fase do ciclo de vida da firma.

Neste contexto, Dickinson et al. (2018) propuseram que o ciclo de vida da firma impacta nos valores patrimoniais. Os autores investigaram o value relevance das informações contábeis e das previsões de lucros por analistas, a partir do estágio de ciclo de vida da firma. Para tanto, seguiram os trabalhos de Collins, Maydew e Weiss (1997) e Schaberl (2016) para decomposição do poder explicativo das regressões de retorno e preço e a proxy de Dickinson (2011) para identificação dos estágios do ciclo de vida. Os principais achados evidenciaram que as informações contábeis e as previsões de lucros dos analistas são relevantes para mensurar o valor de mercado, mas de formas díspares, condicionais ao estágio do ciclo de vida da firma.

Com base em sua pesquisa sobre o ciclo de vida corporativo em contabilidade, finanças e governança corporativa, Habib e Hasan (2019), afirmam que o ciclo de vida da firma gera efeitos significativos na gestão das firmas e são fatores determinantes da competitividade. Entretanto, observaram que, muitos dos estudos neste campo são conceituais e não empíricos. E acrescentam, que estudos que combinam contabilidade e finanças superam essa limitação, desenvolvendo métricas baseadas em contabilidade para categorizar os estágios do ciclo de vida e explorando os antecedentes e as implicações da transição das firmas ao longo dos estágios do ciclo de vida. 
No Brasil, Lima, Carvalho, Paulo e Girão (2015) analisaram para o período de 1995 a 2011, o efeito dos estágios de ciclo de vida das empresas na qualidade das informações contábeis, levando em consideração o conservadorismo, a persistência dos resultados, fluxos de caixa e o gerenciamento dos resultados. Os achados mostraram que há diferenças significativas na qualidade da informação quando considerados os estágios de ciclo de vida das companhias abertas brasileiras. Com relação ao gerenciamento de resultados, os autores concluíram, por exemplo, que empresas em crescimento possuem mais persistência de lucro do que as classificadas no estágio de declínio, e ainda que empresas maduras apresentam mais persistência de lucro que as situadas em outros estágios.

Já Costa, Macedo, Yokoyama e Almeida (2017) analisaram a relação entre os estágios do ciclo de vida das empresas, de acordo com o proposto por Dickinson (2011), e indicadores contábeis e financeiros (distribuição de dividendos, alavancagem, market-to-book, rentabilidade sobre o patrimônio líquido, tamanho da empresa e crescimento das vendas) no período de 2005 a 2012. Os autores evidenciaram que os referidos indicadores podem ser utilizados para a classificar as empresas brasileiras nos estágios de ciclo de vida.

Assim, uma vez que os estágios de ciclo de vida apresentam um papel relevante nas pesquisas mencionadas, pode-se esperar que modelos de previsão de retorno e preço dos ativos, condicionados a essa informação, demonstrem melhor desempenho e auxiliem no entendimento das características subjacentes dos ativos sob análise. Portanto, o foco do presente estudo é investigar se o modelo de avaliação contábil desenvolvido por Lyle et al. (2013) é afetado pelo estágio de ciclo de vida das firmas utilizadas na estimação.

\section{PROCEDIMENTOS METODOLÓGICOS}

\subsection{Amostra}

A amostra foi formada por firmas brasileiras de capital aberto. Os dados relativos aos preços ajustados, quantidade outstanding das ações, valor contábil do patrimônio líquido, lucro líquido, dividendos pagos aos acionistas, previsões de lucro de consenso dos analistas, bem como os fluxos de caixa (operacional, de investimentos e financiamentos) para desenvolver os estágios do ciclo de vida das firmas foram coletados entre os anos de 2010 a 2016. Para os dados contábeis na amostra, as informações em dezembro de t-1 foram utilizadas para as estimações do mês de abril a março do ano $t+1$ sendo assim, as regressões foram realizadas mês a mês.

Seguindo os critérios de seleção de Lyle et al. (2013), as análises foram restritas as firmas com valores contábeis positivos, preço por ação superior a R \$ 5,00, em pelo menos 2 anos consecutivos, previsões de analistas para $\mathrm{t}+1$ e t+2 e as relações book-to-market definidas entre 0,01 e 100. Em Lyle et al. (2013), que seguiu a metodologia de Nekrasov e Shroff (2009), foi necessário que a previsão dos analistas para o segundo ano fosse positiva, bem como que os lucros esperados em crescimento estivesse entre 0 e $100 \%$, entretanto não se considerou esse último filtro no presente estudo, pois Lyle et al. (2013) mostram que não influenciou os resultados das estimações e com isso a amostra se torna maior. Para coleta desses dados utilizou-se a base da Bloomberg, já para os fatores de risco nos modelos de apreçamento que foram incorporados na análise usou-se a base da Quantum.

O Modelo de Lyle et al. (2013) faz uso do risco sistêmico agregado, utilizando como proxy o Volatility Index (VIX), já para esse estudo foi usado o IVol-BR que é disponibilizado no site do Núcleo de Pesquisa em Economia Financeira (NEFIN) da Faculdade de Economia, Administração e Contabilidade da Universidade de São Paulo (FEA/USP), (http://nefin.com.br/volatility index.html). Os dados foram winsorizados a 1\% e 99\%, visando mitigar a influência de outliers.

\subsection{O modelo de Lyle et al. (2013)}

O Modelo de Lyle et al. (2013) está ancorado na teoria de precificação de ativos e é formulado a partir de uma contabilidade imparcial. Assim, em termos de expectativa a taxa de retorno do ativo converge para o custo de capital subjacente. Lyle et al. (2013) relacionam em termos teóricos e empíricos retornos esperados com os custos de capital, mostrando uma dinâmica entre eles. 
Neste artigo, adotou-se a metodologia de Lyle et al. (2013) por considerar os lucros futuros esperados como um dos determinantes para explicar o retorno esperado. Além disso, Lyle et al. (2013) afirmam que a medida é considerada superior aos modelos convencionais, por exemplo, os que se fundamentam no modelo de 3-fatores de Fama e French (1993) (FF) ou no Capital Asset Pricing Model (CAPM).

Finalmente, a metodologia é de fácil aplicação, por gerar retornos esperados apenas como uma combinação linear de variáveis contábeis observáveis. Evans, Njoroge e Yong (2017) afirmam que o modelo é empiricamente implementável e permite comparar diretamente os retornos esperados implícitos por previsões baseadas em modelos e previsões de analistas.

Especificamente, o modelo de Lyle et al. (2013) demonstra que os retornos esperados (custo de capital) da firma podem ser explicitados como uma função linear de variáveis contábeis:

$$
\mu_{t+1}=1+\eta_{1} \frac{x_{L}^{a}}{S_{t}}+\eta_{2} \frac{B_{t}}{S_{t}}+\eta_{3} \frac{x_{t}}{S_{t}}+\eta_{4} \frac{E_{t}\left[x_{t+1}\right]}{S_{t}}+\eta_{5} \frac{D_{t}}{S_{t}}
$$

Em que: $\eta_{1}, \eta_{2} \geq 0 ; \eta_{3} \leq 0 ; \eta_{4}>0 ; \eta_{5} \geq 0$

A Equação 1 representa uma das mais relevantes conclusões teóricas de Lyle et al. (2013), já que implica em considerações para medir o retorno esperado (custo de capital) $\left(\mu_{t+1}\right)$, a partir de uma função linear não-negativa da relação lucros anormais futuros $\left(x_{L}^{a}\right)$, patrimônio líquido $\left(B_{t}\right)$, lucro atual $\left(x_{t}\right)$, expectativas de lucros $E_{t}\left[x_{t+1}\right]$ e dividendos $\left(D_{t}\right)$, em que as variáveis estão em função do preço atual $\left(S_{t}\right)$. Os autores assumem que lucros anormais futuros são transversalmente constantes, portanto, no longo prazo, uma firma crescerá até se assemelhar ao crosssection das empresas.

Callen (2016) avalia que o custo de capital da firma em função de indicadores fundamentalistas já é estudado há muito tempo na literatura de finanças, contudo o estudo de Lyle et al. (2013) foi o primeiro a demonstrar a partir de desenvolvimento teórico. O modelo de regressão empírica se assemelha ao apresentado na Equação 1, sendo definido como:

$$
R_{t+1}-1=\alpha+\frac{\eta_{1}}{S_{t}}+\eta_{2} \frac{B_{t}}{S_{t}}+\eta_{3} \frac{x_{t}}{S_{t}}+\eta_{4} \frac{E_{t}\left[x_{t+1}\right]}{S_{t}}+\eta_{5} \frac{D_{t}}{S_{t}}+\varepsilon_{t+1}
$$

Em que:

$B_{t}=$ Valor Contábil;

$x_{t}=$ Lucros antes de itens extraordinários;

$E_{t}\left[x_{t+1}\right]=$ Expectativa de lucros futuros, que é medida pela média ponderada das previsões de lucro de consenso dos analistas para $\mathrm{t}+1$ e $\mathrm{t}+2$, multiplicada pelas ações ordinárias em circulação;

$D_{t}=$ Dividendos pagos aos acionistas ordinários;

$S_{t}=$ Preço das ações do último período ajustado para stock splits multiplicado pelo número de ações em circulação (representa o tamanho da firma).

Espera-se que os coeficientes para o inverso do tamanho, $\eta_{1}$, relação book-to-market, $\eta_{2}$, lucro-preço futuro, $\eta_{4}$, e dividend yield, $\eta_{5}$, fossem positivos e o coeficiente da relação lucro-preço corrente, $\eta_{3}$, negativo, assim como ocorre em Lyle et al. (2013). As regressões mensais de Fama e MacBeth (1973) foram utilizadas para calcular os coeficientes de cada uma das variáveis. Essas análises foram realizadas para verificar se a equação baseada em fundamentos consegue capturar a dinâmica dos retornos esperados de acordo com o estágio de ciclo de vida das firmas.

Com o propósito de determinar se a sensibilidade ao risco em toda a economia é um fator de risco razoável para a amostra, Lyle et al. (2013) realizaram um teste de precificação de ativos e regrediram os retornos das ações sobre esse fator de risco estimado (deflacionado pelo preço). Além disso, compararam o fator de risco sensibilidade ao risco em toda a economia com o CAPM e com os 3-fatores de FF. Aqui foram retomadas as mesmas análises, porém, considerando o efeito do estágio do ciclo de vida da firma. Para tanto, foi necessário obter essa medida de sensibilidade ao risco de toda a economia. A Equação 3 apresenta a regressão estimada: 


$$
R_{t+1}-R_{f}=\lambda_{0}+\lambda_{1}\left[\left(R_{f}-1\right) \frac{V I X_{t}}{S_{t}}-\frac{\Delta V I X_{t}}{S_{t}}\right]+e_{r, t+1}
$$

Em que $\lambda_{1}$ representa a sensibilidade dos retornos da firma e $e_{r, t+1}$ é o termo de erro com média zero que contém os choques de fluxo de caixa. O VIX é a proxy empírica para o risco esperado em toda a economia (sistêmico) e, no estudo do Lyle et al. (2013) é disponibilizado pela Chicago Board Options Exchange (CBOE), para o mercado americano. Uma vez que o risco de toda a economia é transversalmente constante, a abordagem de duas passagens foi seguida.

Na primeira passagem, a Equação 3 foi estimada a partir de dados de séries temporais no nível da firma. Seguindo os procedimentos de Lyle et al. (2013) a amostra desse estudo foi limitada a ativos com no mínimo 120 dias de negociação. Objetivando minimizar questões de microestrutura, as quais ocorrem com o uso de dados diários, assim como no estudo original, foi acrescentada a variável independente defasada nas estimações. $\mathrm{O}$ coeficiente de regressão, $\lambda_{1}$, mede a sensibilidade dos retornos da firma para o risco em toda a economia. Já a sua estimativa, é o somatório dos coeficientes nas variáveis independentes atual e defasada, que representa uma métrica de sensibilidade atrelada ao risco da firma.

Em seguida, na segunda passagem da regressão em corte transversal, foram regredidos os retornos excedentes no coeficiente de sensibilidade estimado para o risco agregado. Assim, incorporou-se, a estimativa de $\lambda_{1}$, como uma variável adicional na regressão em corte transversal de segunda passagem. O IVol-BR do NEFIN possui dados apenas a partir de agosto de 2011, portanto foi utilizada uma janela de 3 anos para mensurar as sensibilidades, visando ser condizente com o período usado no primeiro e no segundo estágio. Foram incluídos testes com os modelos de apreçamento amplamente usados na literatura, o CAPM e o modelo de 3-fatores de FF. Uma abordagem similar de regressão de duas passagens é seguida para estimar as sensibilidades para o beta do CAPM e os betas do modelo de 3-fatores de FF durante o mesmo período de tempo.

Adicionalmente, outro fator de risco denominado Cross-Sectional Variance (CSV) foi investigado para averiguar se seria relevante para as estimações nos diferentes estágios de ciclo de vida das firmas. De acordo com Goyal e Santa-Clara (2003) e Garcia, Mantilla-García e Martellini (2014) a CSV pode ser uma proxy relevante para o risco idiossincrático agregado, já que nos Estados Unidos da América (EUA) viu-se que a CSV dos retornos das ações é correlacionada com os retornos de mercado. Os autores descobriram que ações com alta sensibilidade à dispersão (CSV) oferecem baixos retornos esperados. Concluíram também que uma carteira spread a custo zero que é long (short) em ações com betas de baixa (alta) dispersão produziu um retorno estatisticamente e economicamente significativo e, encontraram que a dispersão está associada a um prêmio de risco significativamente negativo.

Para este estudo, a CSV foi desenvolvida usando como base Garcia et al. (2014). Considerando a CSV ponderada em igualdade, a seguir denominada $C S V_{t}^{E W}$ em que $w_{i t}=1 / N_{t} \forall i$ e $t$. Assumindo que $r_{t}^{E W}$ representa o retorno do portfólio igualmente ponderado, tem-se:

$$
\operatorname{CSV}_{t}^{E W}=\frac{1}{N_{t}} \sum_{i=1}^{N_{t}}\left(r_{i t}-r_{t}^{E W}\right)^{2}
$$

Em que:

$N_{t}=$ número de ações em um portfólio;

$i=1,2, \ldots, N_{t}$

$r_{i t}=$ retorno de cada ação.

Com relação às questões de microestrutura que podem ocorrer utilizando dados diários, do mesmo modo que foi feito com o IVol-BR e os fatores de risco, incluiu-se também a variável independente defasada da $C S V^{E W}$ na mensuração.

Assim como Lyle et al. (2013), procurou-se verificar também se o modelo possui desempenho satisfatório em prever os preços das ações para o mercado de capitais brasileiro, porém considerando o estágio do ciclo de vida da firma. Os autores sugerem, para o mercado americano, que os fundamentos das firmas são positivamente correlacionados com os preços dos ativos, entretanto inversamente relacionados com o risco de toda a economia.

$\mathrm{Na}$ Equação 5, para estimação dos preços, as variáveis foram deflacionadas em ambos os lados pelo valor contábil do patrimônio e a substituição do risco em toda a economia, pelo VIX. Como segue: 


$$
\frac{S_{t}}{B_{t}}=\frac{y_{1}}{B_{t}}+y_{2}+y_{3} \frac{x_{t}}{B_{t}}+y_{4} \frac{D_{t}}{B_{t}}+\frac{E_{t}\left[x_{t+1}\right]}{B_{t}}-\frac{\lambda e s t_{1} V I X_{t}}{B_{t}}+w_{t+1}
$$

Em que $w_{t+1}$ é um termo de erro com média zero.

Para análise do ciclo de vida, este artigo seguiu a metodologia de Dickinson (2011) com o objetivo de investigar se as alterações que ocorrem com o passar do tempo na firma impactam a previsão de retornos e de preços, por meio de diferentes estágios do ciclo de vida da firma.

Neste sentido, os fluxos de caixa seguem padrões de acordo com suas atividades operacionais, de investimentos e financiamentos para cada ciclo. Dickinson (2011) argumenta que o fluxo de caixa captura diferenças na lucratividade, crescimento e risco da firma, assim é possível usar o fluxo de caixa operacional (FCO), de investimento (FCI) e de financiamento (FCF) para agrupar firmas em estágios do ciclo de vida. Portanto, seguindo esse raciocínio este estudo utilizou a referida metodologia como segue: introdução, se $\mathrm{FCO}<0, \mathrm{FCI}<0$ e FCF $>0$; crescimento, se FCO $>0$, FCI $<0$ e FCF $>0$; maturidade, se FCO $>0$, FCI $<0$ e FCF $<0$; declínio, se $\mathrm{FCO}<0, \mathrm{FCI}>0$ e FCF $\leq$ ou $\geq 0$; e os demais anos das firmas são classificados no estágio de turbulência.

Contudo, como a série temporal não é longa, ocasionando poucas informações em alguns estágios do ciclo de vida das firmas, posteriormente houve a junção de alguns estágios para que a amostra fosse mais consistente. Assim, as análises foram feitas levando em consideração: i) amostra completa; ii) firmas em crescimento; iii) firmas maduras; iv) junção das firmas em crescimento e maduras; e v) demais ciclos, com exceção das firmas em crescimento e maduras.

\section{ANÁLISE E DISCUSSÃO DOS RESULTADOS}

De acordo com Hasan et al. (2015) as firmas grandes e maduras são ricas, diversificadas e possuem maior força de mercado, enquanto as menores e mais jovens firmas são o oposto. Portanto as firmas maduras conseguem obter vantagem competitiva consistentemente e reduzem os riscos para os investidores.

Neste contexto, deveriam apresentar retornos mais baixos e preços mais altos de suas ações, mas os resultados mostram que no Brasil as firmas maduras geram os maiores retornos, lucros e dividendos pagos e que exibem menores preços, valores contábeis do patrimônio líquido, lucros previstos pelos analistas e relação bookto-market. Uma possível explicação para tal resultado pode ser a existência de caraterísticas distintas do mercado brasileiro em relação a economias mais desenvolvidas como, por exemplo, alta concentração de propriedade, menor cobertura de analistas, fatores macroeconômicos específicos, alta participação do Estado na economia e menor quantidade de dados disponíveis para análises.

Já o estudo de Costa et al. (2017) mostrou que empresas maduras possuem maiores dividendos, retorno sobre o PL e tamanho se comparado com as empresas classificadas como no estágio de introdução, sendo este um resultado similar ao que ocorreu no presente trabalho quanto aos dividendos e a variável tamanho.

As firmas, com exceção das classificadas no estágio de crescimento e maduras, foram as que apresentaram menores retornos e por serem mais arriscadas deveriam oferecer resultado oposto. Além disso, são as com maiores preços, valores contábeis do patrimônio, lucros previstos pelos analistas e relação book-to-market.

Estas firmas também exibem altas variações nos resultados, representados pelos desvios-padrão na Tabela 1. De forma geral firmas maduras apresentaram menores desvios, já que normalmente estão consolidadas no mercado e tem maior constância nos resultados. Ainda, como apresentado na Tabela 1, verificou-se que todas as relações book-to-market são negativamente correlacionadas com os retornos esperados. 
Tabela 1. Estatísticas Descritivas

\begin{tabular}{|c|c|c|c|c|c|c|c|c|}
\hline & $R_{t+1}$ & $P_{t}$ & $B_{t}$ & $x_{t}$ & $E_{t}\left[x_{t+1}\right]$ & $D / S_{t}$ & $\operatorname{Tam}_{t}$ & $b m_{t}$ \\
\hline \multicolumn{9}{|c|}{ Painel A: Ciclo de vida: Amostra completa } \\
\hline Média & 0,9987 & 26,581 & 22,884 & 1,038 & 2,065 & 0,033 & 22,326 & $-0,578$ \\
\hline Desvio Padrão & 0,104 & 48,707 & 45,868 & 4,501 & 3,577 & 0,040 & 1,248 & 1,023 \\
\hline Máximo & 1,288 & 411,650 & 274,474 & 23,761 & 26,906 & 0,247 & 25,589 & 1,630 \\
\hline Mínimo & 0,713 & 5,233 & 0,496 & $-26,052$ & $-3,732$ & 0,000 & 19,602 & $-3,116$ \\
\hline \multicolumn{9}{|c|}{ Painel B: Ciclo de Vida: firmas em estágio de crescimento } \\
\hline Média & 1,0006 & 24,082 & 20,701 & 1,050 & 1,865 & 0,026 & 22,344 & $-0,575$ \\
\hline Desvio Padrão & 0,105 & 37,653 & 41,236 & 3,856 & 2,674 & 0,035 & 1,239 & 0,985 \\
\hline Máximo & 1,288 & 411,650 & 274,474 & 16,678 & 26,906 & 0,247 & 25,589 & 1,630 \\
\hline Mínimo & 0,713 & 5,233 & 0,496 & $-26,052$ & $-3,732$ & 0,000 & 19,602 & $-3,116$ \\
\hline \multicolumn{9}{|c|}{ Painel C: Ciclo de Vida: firmas em estágio de maturidade } \\
\hline Média & 1,0015 & 20,969 & 15,045 & 1,503 & 1,812 & 0,039 & 22,570 & $-0,728$ \\
\hline Desvio Padrão & 0,097 & 16,928 & 23,454 & 3,031 & 2,309 & 0,040 & 1,229 & 1,052 \\
\hline Máximo & 1,288 & 151,177 & 274,474 & 23,761 & 26,906 & 0,247 & 25,589 & 1,630 \\
\hline Mínimo & 0,713 & 5,233 & 0,496 & $-26,052$ & $-3,732$ & 0,000 & 19,602 & $-3,116$ \\
\hline \multicolumn{9}{|c|}{ Painel D: Ciclo de Vida: firmas em estágios de crescimento e maturidade } \\
\hline Média & 1,0011 & 22,395 & 17,635 & 1,295 & 1,836 & 0,033 & 22,467 & $-0,658$ \\
\hline Desvio Padrão & 0,101 & 28,407 & 32,935 & 3,441 & 2,483 & 0,038 & 1,239 & 1,025 \\
\hline Máximo & 1,288 & 411,650 & 274,474 & 23,761 & 26,906 & 0,247 & 25,589 & 1,630 \\
\hline Mínimo & 0,713 & 5,233 & 0,496 & $-26,052$ & $-3,732$ & 0,000 & 19,602 & $-3,116$ \\
\hline \multicolumn{9}{|c|}{ Painel E: Ciclo de Vida: demais estágios do ciclo de vida (exceto crescimento e maturidade) } \\
\hline Média & 0,9870 & 47,429 & 49,026 & $-0,244$ & 3,206 & 0,031 & 21,627 & $-0,177$ \\
\hline Desvio Padrão & 0,120 & 98,262 & 79,778 & 7,764 & 6,656 & 0,046 & 1,042 & 0,913 \\
\hline Máximo & 1,288 & 411,650 & 274,474 & 23,761 & 26,906 & 0,247 & 24,875 & 1,630 \\
\hline Mínimo & 0,713 & 5,233 & 0,496 & $-26,052$ & $-3,732$ & 0,000 & 19,602 & $-3,116$ \\
\hline
\end{tabular}

Fonte: Elaborada pelos autores.

Nota: A tabela 1 apresenta as estatísticas descritivas para 6.268 ações-mês de 2010 a 2016 para amostra completa e de acordo com os estágios de ciclo de vida das firmas. $t$ indica o ano fiscal mais recente, $R_{t+l}$ é o retorno mensal com dividendos “cum-dividend" (bruto), $P_{t}$ é o preço por ação para o período $t ; S_{t}$ é a quantidade da ação no final do ano fiscal mais recente multiplicada pelo preço no final do mês calendário $t$, $B_{t}$ é o valor contábil do patrimônio líquido no final do ano fiscal $t ; x_{t}$ é o lucro líquido para o ano fiscal $t$; $D t$ são os dividendos pagos aos acionistas no ano fiscal $t ; E_{t}\left[x_{t+1}\right]$ é a expectativa de lucros futuros, que é medida pela média ponderada das previsões de lucro de consenso dos analistas para $t+1$ e $t+2$, multiplicada pela quantidade de ações em circulação no mês $t$; Tam $t_{t}$ é o tamanho das firmas; e bm a relação book-to-market.

Os resultados da matriz de correlação apresentados na Tabela 2 são similares aos apresentados em Lyle et al. (2013), pois há baixa correlação entre a variável dependente $\left(R_{t+1}-1\right)$ e as independentes do modelo, tanto na amostra completa quanto nos modelos estimados em cada estágio do ciclo de vida das firmas.

$\mathrm{Na}$ amostra completa, que não leva em consideração os estágios de ciclo de vida, todas as variáveis com exceção de dividendos possuem correlação com a variável dependente. Sendo que o inverso do tamanho e o valor contábil do patrimônio são negativamente correlacionadas com a referida variável.

Altas correlações, conforme já se esperava, ocorreram entre os lucros e as previsões de lucros dos analistas. Entretanto a maior correlação $(0,647)$ foi entre as variáveis inverso do tamanho e valor contábil do patrimônio. No geral, as correlações entre as estimativas de retornos e os estágios do ciclo de vida foram semelhantes as encontradas por Lyle et al. (2013), sendo que neste último não houve a estratificação da amostra a partir dos ciclos de vida das firmas. 
Tabela 2. Matriz de Correlações de Pearson

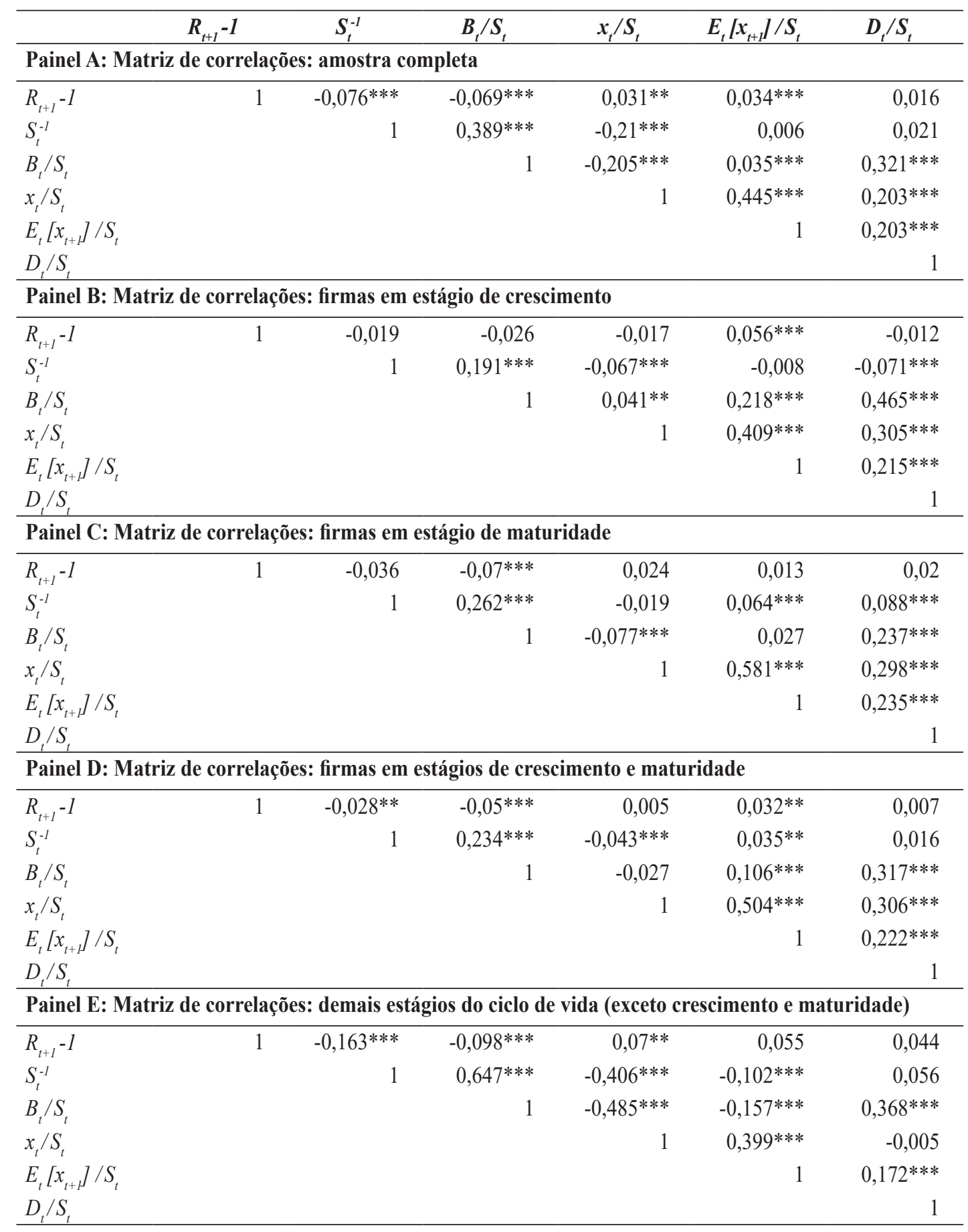

Fonte: Elaborada pelos autores.

Notas: ***, ** e * representam as significâncias a 1\%,5\% e 10\%, respectivamente. A tabela 2 fornece as correlações de Pearson para a variáveis da amostra completa e entre os estágios de ciclo de vida das firmas.

Os resultados contidos na Tabela 3 evidenciam que o modelo contábil não é adequado para o prever retornos esperados no mercado brasileiro, mesmo quando a amostra é subdividida em estágios do ciclo de vida das firmas. Confirma-se também a pouca previsibilidade das estimações pelos baixos $R^{2}$ ajustados. Contudo, na amostra completa com dados do Brasil percebe-se que o valor contábil do patrimônio $\left(B_{t} / S_{t}\right)$ é altamente significativo, possuindo uma relação negativa com os retornos esperados. De forma geral o $B_{t} / S_{t}$ é significativo e relacionado negativamente com firmas que estão no grupo de crescimento e para os grupos de crescimento e maturidade analisados conjuntamente. 
Tabela 3. Regressões cross-section por estágios de ciclo de vida da firma

\begin{tabular}{lccccc}
\hline \multicolumn{5}{c}{ Modelo de regressão: $R_{t+1}-1$} & Intercepto $+\eta_{1} / S_{t}+\eta_{2} B_{t} / S_{t}+\eta_{3} x_{t} / S_{t}+\eta_{t}\left(E_{t}\left[x_{t+1}\right]\right) / S_{t}+\eta_{5} D_{t} / S_{t}$ \\
& $\begin{array}{c}\text { Amostra } \\
\text { completa }\end{array}$ & Crescimento & Maturidade & $\begin{array}{c}\text { Crescimento ou } \\
\text { Maturidade }\end{array}$ & $\begin{array}{c}\text { Exceção, crescimento } \\
\text { ou maturidade }\end{array}$ \\
\hline Intercepto & $-0,039$ & 0,025 & $-0,044$ & $-0,018$ & $-0,179$ \\
& $(-1,247)$ & $(0,529)$ & $(-1,061)$ & $(-0,597)$ & $(-1,254)$ \\
$S_{t}^{-1}$ & $-0,001$ & 0,002 & $-0,002$ & $-0,001$ & $-0,008$ \\
& $(-1,123)$ & $(0,762)$ & $(-1,079)$ & $(-0,403)$ & $(-1,276)$ \\
$B_{t} / S_{t}$ & $-0,009 * * *$ & $-0,013 * * *$ & $-0,007$ & $-0,009 * * *$ & $-0,014 *$ \\
& $(-2,721)$ & $(-2,468)$ & $(-1,515)$ & $(-2,392)$ & $(-1,747)$ \\
$x_{t} / S_{t}$ & 0,022 & 0,025 & 0,022 & 0,019 & 0,076 \\
& $(0,872)$ & $(0,447)$ & $(0,473)$ & $(0,571)$ & $(1,365)$ \\
$E_{t}\left[x_{t+1}\right] / S_{t}$ & 0,013 & $0,127 *$ & $-0,068$ & 0,035 & $-0,114$ \\
& $(0,413)$ & $(1,943)$ & $(-1,176)$ & $(0,892)$ & $(-1,432)$ \\
$D_{t} / S_{t}$ & 0,052 & $-0,083$ & $0,120 *$ & 0,042 & $0,621 * * *$ \\
\hline $\mathrm{R}^{2}$ ajustado & $(0,994)$ & $(-0,750)$ & $(1,736)$ & $(0,768)$ & $(3,098)$ \\
\hline
\end{tabular}

Fonte: Elaborada pelos autores.

Notas: ***,** e representam as significâncias a 1\%, $5 \%$ e $10 \%$, respectivamente. Esta tabela relata os coeficientes médios e as estatísticas t das regressões cross-section de Fama e MacBeth (1973) do excesso de retorno das ações em um mês a frente de acordo com os estágios de ciclo de vida.

Para os estágios do ciclo de vida, com a exceção dos estágios de crescimento e maturidade, $x_{t} / S_{t}$ apresentouse marginalmente significativa, neste mesmo grupo de firmas a variável dividendos foi altamente significativa. Ademais, a variável dividendos foi marginalmente significativa nas firmas em estágio de maturidade e os lucros previstos pelos analistas marginalmente significativos nas firmas em estágio de crescimento.

A regressão 1 da Tabela 4 evidenciou que a sensibilidade ao risco em toda a economia, $\lambda$ est $/ S_{t}$, não possui significância estatística, diferente do que ocorreu em Lyle et al. (2013), sugerindo que este fator de risco não deve ser usado para a precificação do risco no país em análise, mesmo quando as firmas são classificadas a partir de diferentes estágios do ciclo de vida.

Em Lyle et al. (2013) os betas do CAPM (regressão 2 - painel E) e de 3-fatores (regressão 3 - paineis A e E) foram marginalmente significantes, no Brasil os resultados apresentam evidências semelhantes. Para os ciclos de vida, com exceção das firmas em crescimento e maduras, a regressão 4 - painel E, a Cross-Sectional Variance (CSV) apresentou significância marginal, ou seja, pode ser um fator de risco importante para firmas classificadas nesses estágios. 
Tabela 4. Regressões de retorno cross-section e estimativas de fatores de risco em diferentes estágios do ciclo de vida da firma

\begin{tabular}{|c|c|c|c|c|}
\hline & Regressão 1 & Regressão 2 & Regressão 3 & Regressão 4 \\
\hline \multicolumn{5}{|c|}{ Painel A: Estimativas de fatores de risco: amostra completa } \\
\hline \multirow[t]{2}{*}{$\lambda e s t / S_{t}$} & $-60,355$ & & & \\
\hline & $(-1,620)$ & & & \\
\hline \multirow[t]{2}{*}{$\beta$} & & 0,000 & & \\
\hline & & $(0,007)$ & & \\
\hline \multirow[t]{2}{*}{$\beta_{m}$} & & & $0,005^{* *}$ & \\
\hline & & & $(0,216)$ & \\
\hline \multirow[t]{2}{*}{$\beta_{s}$} & & & $-0,024 * *$ & \\
\hline & & & $(-2,274)$ & \\
\hline \multirow[t]{2}{*}{$\beta_{h}$} & & & $-0,004$ & \\
\hline & & & $(-0,199)$ & \\
\hline \multirow[t]{2}{*}{$C S V^{E W}$} & & & & 0,003 \\
\hline & & & & $(0,426)$ \\
\hline $\mathrm{R}^{2}$ ajustado & 0,014 & 0,075 & 0,122 & 0,008 \\
\hline
\end{tabular}

\section{Painel B: Estimativas de fatores de risco: firmas em estágio de crescimento}

\begin{tabular}{|c|c|c|c|}
\hline$\lambda e s t / S_{t}$ & $\begin{array}{c}-29,953 \\
(-0,410)\end{array}$ & & \\
\hline$\beta$ & & $\begin{array}{l}0,021 \\
(0,791)\end{array}$ & \\
\hline$\beta_{m}$ & & & $\begin{array}{l}0,029 \\
(0,794)\end{array}$ \\
\hline$\beta_{s}$ & & & $\begin{array}{l}0,003 \\
(0,200)\end{array}$ \\
\hline$\beta_{h}$ & & & $\begin{array}{l}0,008 \\
(0,257)\end{array}$ \\
\hline
\end{tabular}

$\begin{array}{ll}C S V^{E W} & 0,005\end{array}$

\begin{tabular}{|c|}
\hline $\mathrm{R}^{2}$ ajustado \\
\hline
\end{tabular}

Painel C: Estimativas de fatores de risco: firmas em estágio de maturidade

$\lambda$ est $/ S_{t} \quad-50,924$

$(-0,880)$

$\beta \quad-0,033$

$(-0,964)$

$\beta_{m}$

$\beta_{s}$

$\beta_{h}$

$(-0,352)$

CSVEW

Fonte: Elaborada pelos autores.

Notas: $* * *, * * \mathrm{e} *$ representam as significâncias a 1\%,5\% e 10\%, respectivamente. A tabela apresenta os coeficientes médios e as estatísticas t da regressão cross-section de Fama e MacBeth (1973) do retorno excedente um mês a frente sobre os fatores de risco estimados, são eles: $\left(\lambda\right.$ est $\left./ S_{t}\right)$ é a sensibilidade específica da firma para o risco agregado, $(\beta)$ é o beta do CAPM e $\left(\beta_{m}, \beta_{s}, \beta_{h}\right)$ são os betas do modelo de 3-fatores de FF e $C S V^{E W}$ é a Cross-Sectional Variance usando um esquema de peso igualmente ponderado para a amostra completa e para os estágios de ciclo de vida. 
Tabela 4. Regressões de retorno cross-section e estimativas de fatores de risco em diferentes estágios do ciclo de vida da firma (continuação)

\begin{tabular}{|c|c|c|c|c|}
\hline & Regressão 1 & Regressão 2 & Regressão 3 & Regressão 4 \\
\hline \multicolumn{5}{|c|}{ Painel D: Estimativas de fatores de risco: firmas em estágios de crescimento e maturidade } \\
\hline$\lambda e s t / S_{t}$ & $\begin{array}{l}-52,665 \\
(-1,198)\end{array}$ & & & \\
\hline$\beta$ & & $\begin{array}{l}-0,011 \\
(-0,383)\end{array}$ & & \\
\hline$\beta_{m}$ & & & $\begin{array}{l}-0,014 \\
(-0,555)\end{array}$ & \\
\hline$\beta_{s}$ & & & $\begin{array}{l}-0,017 \\
(-1,214)\end{array}$ & \\
\hline$\beta_{h}$ & & & $\begin{array}{l}-0,003 \\
(-0,135)\end{array}$ & \\
\hline$C S V^{E W}$ & & & & $\begin{array}{l}-0,001 \\
(-0,119)\end{array}$ \\
\hline $\mathrm{R}^{2}$ ajustado & 0,015 & 0,098 & 0,154 & 0,009 \\
\hline \multicolumn{5}{|c|}{ Painel E: Estimativas de fatores de risco: demais estágios do ciclo de vida (exceto crescimento e maturidade) } \\
\hline$\lambda e s t / S_{t}$ & $\begin{array}{l}19,360 \\
(0,287)\end{array}$ & & & \\
\hline$\beta$ & & $\begin{array}{l}0,083 * * * \\
(2,480)\end{array}$ & & \\
\hline$\beta_{m}$ & & & $\begin{array}{l}0,147 * * * \\
(3,316)\end{array}$ & \\
\hline$\beta_{s}$ & & & $\begin{array}{l}-0,079^{* * *} \\
(-2,871)\end{array}$ & \\
\hline$\beta_{h}$ & & & $\begin{array}{l}0,025 \\
(0,800)\end{array}$ & \\
\hline$C S V^{E W}$ & & & & $\begin{array}{l}0,040^{*} \\
(1,843)\end{array}$ \\
\hline $\mathrm{R}^{2}$ ajustado & 0,063 & 0,037 & 0,128 & 0,074 \\
\hline
\end{tabular}

Fonte: Elaborada pelos autores.

Notas: ***,** e* representam as significâncias a 1\%, 5\% e 10\%, respectivamente. A tabela apresenta os coeficientes médios e as estatísticas t da regressão cross-section de Fama e MacBeth (1973) do retorno excedente um mês a frente sobre os fatores de risco estimados, são eles: $\left(\lambda e s t / S_{t}\right)$ é a sensibilidade específica da firma para o risco agregado, $(\beta)$ é o beta do CAPM e $\left(\beta_{m}, \beta_{s}, \beta_{h}\right)$ são os betas do modelo de 3 -fatores de FF e $C S V^{E W}$ é a Cross-Sectional Variance usando um esquema de peso igualmente ponderado para a amostra completa e para os estágios de ciclo de vida.

A Tabela 5 sugere que o modelo contábil se mostrou relevante para previsão de preços no mercado de capitais brasileiro em diferentes estágios do ciclo de vida das firmas. Com base na hipótese de prices lead earnings a informação contida nos preços é mais completa em relação aos lucros contábeis atuais e passados para a previsão de lucros (Kothari, 2001). De acordo com o $\mathrm{R}^{2}$ ajustado da Tabela 5 o modelo contábil prevê melhor os preços das firmas maduras $\mathrm{R}^{2}=0,821$, contudo com exceção da variável lucros, que não apresentou significância, todas as variáveis são significantes a $1 \%$. Já o menor $\mathrm{R}^{2}=0,691$, foi para firmas no estágio de crescimento, que também não exibiu significância para os lucros.

De forma geral, os lucros só foram significantes a um nível de 5\% para os estágios do ciclo de vida, com exceção das firmas em estágio de crescimento e maduras. Ressalta-se que para as firmas nestes estágios o intercepto, o inverso do tamanho, os lucros e dividendos são negativamente relacionados com os preços, apenas os lucros previstos pelos analistas têm correlação positiva com os preços. 
Tabela 5. Regressões cross-section para previsão de preços

\begin{tabular}{lccccc}
\hline \multicolumn{5}{c}{ Modelo de regressão: $S_{t} / B_{t}=\gamma_{1} / B_{t}+\gamma_{2}+\gamma_{3} x_{t} / B_{t}+\gamma_{4} D_{t} / B_{t}+\gamma_{5}\left(E_{t}\left[x_{t+1}\right]\right) / B_{t}-\lambda e s t_{1} V I X_{t} / B_{t}$} \\
& $\begin{array}{c}\text { Amostra } \\
\text { completa }\end{array}$ & Crescimento & Maturidade & $\begin{array}{c}\text { Crescimento ou } \\
\text { Maturidade }\end{array}$ & $\begin{array}{c}\text { Exceção, crescimento } \\
\text { ou maturidade }\end{array}$ \\
\hline Intercepto & $4,935^{* * *}$ & $3,672^{* * *}$ & $6,257^{* * *}$ & $5,369^{* * *}$ & $-7,390^{* *}$ \\
& $(9,519)$ & $(2,976)$ & $(9,729)$ & $(7,583)$ & $(-2,023)$ \\
$S_{t}^{-1}$ & $0,195^{* * *}$ & $0,147 * * *$ & $0,248^{* * *}$ & $0,212^{* * *}$ & $-0,357^{* *}$ \\
& $(9,759)$ & $(2,855)$ & $(9,779)$ & $(7,654)$ & $(-2,234)$ \\
$x_{t} / S_{t}$ & $0,794 * * *$ & 0,732 & 0,246 & 0,490 & $-2,305^{* *}$ \\
& $(2,649)$ & $(0,862)$ & $(0,356)$ & $(1,238)$ & $(-2,184)$ \\
$E_{t}\left[x_{t+1}\right] / S_{t}$ & $3,542^{* * *}$ & $3,257^{* * *}$ & $3,817 * * *$ & $3,255^{* * *}$ & $10,265^{* * *}$ \\
$D_{t} / S_{t}$ & $(7,226)$ & $(6,305)$ & $(4,472)$ & $(6,543)$ & $(6,089)$ \\
$\mathrm{R}^{2}$ ajustado & $11,317 * * *$ & $16,598^{* * *}$ & $11,095^{* * *}$ & $12,320 * * *$ & $-1,333^{* * *}$ \\
\hline
\end{tabular}

Fonte: Elaborada pelos autores.

Notas: $* * *, * *$ e $*$ representam as significâncias a $1 \%, 5 \%$ e $10 \%$, respectivamente. A tabela apresenta os coeficientes e as estatísticas t das regressões cross-section de Fama e MacBeth (1973), onde a razão preço pelo valor patrimonial foi a variável dependente. $S_{t}$ é o preço ajustado por ação multiplicado por ações em circulação, $B_{t}$ é o valor contábil do patrimônio, $x_{t}$ são os lucros atuais e $D_{t}$ dividendo por ação. $E_{t}\left[x_{t+t}\right]$ é a expectativa de lucros futuros, que é medida pela média ponderada das previsões de lucro de consenso dos analistas para $t+1$ e $t+2$, multiplicada pela quantidade de ações em circulação no mês $t$. hest $t_{l}$ é a sensibilidade específica da firma para o risco agregado. As variáveis foram analisadas de forma conjunta e de acordo com os ciclos de vida das firmas. As estatísticas t são calculadas a partir das regressões de Fama e MacBeth (1973).

Percebe-se que o modelo tem boa adequação para prever preços das firmas em estágio de crescimento e maduras conjuntamente $\left(\mathrm{R}^{2}=0,802\right)$, entretanto a variável representativa dos lucros não exibiu significância estatística. O terceiro maior $\mathrm{R}^{2}(0,794)$ foi para as estimações com a amostra completa e todas as variáveis foram significativas, mostrando assim que a mensuração para previsão de preços no Brasil é relevante. Entre os coeficientes calculados o relativo aos dividendos possui os maiores valores nos diferentes ciclos de vida das firmas, assim pode-se inferir que é a variável com maior peso na previsão de preços no país.

\section{CONCLUSÕES}

No presente estudo, analisou-se a influência dos diferentes estágios do ciclo de vida das firmas, conforme metodologia de Dickinson (2011), na previsão de retornos e preços a partir do modelo de Lyle et al. (2013). Os resultados indicaram que o modelo proposto não tem capacidade preditiva para previsão de retornos usando dados brasileiros, mesmo quando a amostra é dividida com base em diferentes estágios do ciclo de vida das firmas. Lyle et al. (2013) não consideraram ciclos de vida em sua análise, mas os seus achados para o mercado americano são contrastantes com o que ocorreu no mercado brasileiro em relação aos retornos.

Já para as estimações de preços visualiza-se que o modelo possui boa adequação e as variáveis de forma geral são significativas para explicação da variável dependente $\left(S_{t} / B_{t}\right)$. Percebe-se que a metodologia prevê melhor os preços das firmas maduras, como pode ser visto pelo $\mathrm{R}^{2}$ ajustado de 0,821 e para as firmas em crescimento reportou-se o menor $\mathrm{R}^{2}(0,691)$. Com base no que foi analisado, os ciclos de vida das firmas afetam as estimações no Brasil de diferentes formas, o que contribui para literatura, por exemplo, complementando o trabalho de Hasan e Habib (2017), já que foi evidenciado que há variações, mesmo que não sejam altamente significativas, no desempenho e nos riscos as quais são condicionadas pelos estágios dos ciclos de vida para firmas brasileiras.

Entre as limitações deste estudo, a principal pode ser definida como o tamanho da amostra considerado pequeno e a abrangência dos anos analisados. Entretanto, tentou-se que os dados refletissem o mercado de capitais brasileiro a partir da diversidade das firmas pesquisadas. Como sugestões para trabalhos futuros recomenda-se investigar os efeitos das multidimensões da competição do mercado produtos para verificar se interferem na capacidade do modelo de avaliação contábil em prever os retornos e preços das ações no mercado de capitais brasileiros, já que segundo Sharma (2011) os temas estão relacionados e levam em consideração as características do ambiente em que as firmas se inserem. 


\section{REFERÊNCIAS}

Antônio, R. M., Lima, F. G., \& Pimenta Junior, T. (2015). Stock Recommendations and Investment Portfolio Formation: A Study in the Brazilian Market. Contaduría y Administración, 60(4): 874-92. DOI: https://doi. org/10.1016/j.cya.2015.07.010.

Antônio, R. M., Ambrozini, L. C. S., Gatsios, R. C., \& Magnani, V. M. (2017). Analysts' Consensus and Target Price Accuracy: A Study in Latin America. Brazilian Administration Review - BAR, 14(4), 1-19. DOI: http:// dx.doi.org/10.1590/1807-7692bar2017170036.

Antônio, R. M., Lima, F. G., dos Santos, R. B., \& Rathke, A. A. T. (2019). Use of Derivatives and Analysts' Forecasts: New Evidence from Non-financial Brazilian Companies. Australian Accounting Review, 29(1), 220-234. DOI: https://doi.org/10.1111/auar.12268.

Callen, J. L. (2016). Accounting Valuation and Cost of Equity Capital Dynamics. Abacus, 52(1): 5-25. DOI: http:// dx.doi.org/10.1111/abac.12070.

Collins, D., Maydew, E., \& Weiss, I. (1997). Changes in the value-relevance of earnings and book values over the past forty years. Journal of Accounting and Economics, 24(1): 39-67. DOI: https://doi.org/10.1016/S01654101(97)00015-3.

Costa, W. B., Macedo, M. A. S., Yokoyama, K. Y., \& Almeida, J. E. F. (2017). Análise dos Estágios de Ciclo de Vida de Companhias Abertas no Brasil: Um Estudo com Base em Variáveis Contábil-Financeiras. Brazilian Business Review - BBR, 14(3): 304-320. DOI: http://dx.doi.org/10.15728/bbr.2017.14.3.3.

DeAngelo, H., DeAngelo, L., \& Stulz, R. M. (2006). Dividend Policy and the Earned/Contributed Capital Mix: A Test of the Lifecycle Theory. Journal of Financial Economics, 81(2): 227-254. DOI: http://dx.doi.org/10.2139/ ssrn.766086.

Dickinson, V. (2011). Cash Flow Patterns as a Proxy for Firm Life Cycle. The Accounting Review, 86(6): 19691994. DOI: https://doi.org/10.2308/accr-10130.

Dickinson, V., Kassa, H., \& Schaberl, P. D. (2018). What information matters to investors at different stages of a firm's life cycle? Advances in Accounting, 42, 22-33. DOI: https://doi.org/10.1016/j.adiac.2018.07.002.

Evans, M. E., Njoroge, K., \& Yong, K. O. (2017). An Examination of the Statistical Significance and Economic Relevance of Profitability and Earnings Forecasts from Models and Analysts. Contemporary Accounting Research, 34(3): 1453-1488. DOI: https://doi.org/10.1111/1911-3846.12307.

Fama, E., \& French, K. (1993). Common risk factors in the returns on stocks and bonds. Journal of Financial Economics, 33(1): 3-56. DOI: https://doi.org/10.1016/0304-405X(93)90023-5.

Fama, E., \& Macbeth, J. D. (1973). Risk, return, and equilibrium: Empirical tests. Journal of Political Economy, 81(3): 607-636. Disponível em: http://www.jstor.org/stable/1831028.

Faff, R., Kwok, W. C., Podolski, E. J., \& Wong, G. (2016). Do corporate policies follow a life-cycle? Journal of Banking \& Finance, 69, 95-107. DOI: http://dx.doi.org/10.2139/ssrn.2610483.

Feltham, G., \& Ohlson, J. A. (1999). Residual earnings valuation with risk and stochastic interest rates. The Accounting Review, 74(2): 165-183. Disponível em: www.jstor.org/stable/248579.

Feltham, G., \& Ohlson, J. A. (1995). Valuation and clean surplus accounting for operating and financial activities. Contemporary Accounting Research, 11(2): 689-731. DOI: https://doi.org/10.1111/j.1911-3846.1995. tb00462.x.

Garcia, R., Mantilla-García, D., \& Martellini, D. (2014). A model-free measure of aggregate idiosyncratic volatility and the prediction of market returns. Journal of Financial and Quantitative Analysis, 49(5-6): 1133-1165. DOI: https://doi.org/10.1017/S0022109014000489.

Gatsios, R. C., \& Lima, F. G. (2014). Forecast Bias of Estimates of Market Analysts in the Brazilian Capital Market: Impact of the IFRS Adoption. Business and Management Review, 3(11), 1-6.

Goyal, A., \& Santa-Clara, P. (2003). Idiosyncratic Risk Matters! Journal of Finance, 58(3): 975-1008. Disponível em: www.jstor.org/stable/3094569.

Hasan, M. M., \& Cheung, A. W-K. (2018). Organization capital and firm life cycle. Journal of Corporate Finance, 48, 556-578. Disponível em: https://ssrn.com/abstract=2483834. 
Habib, A., \& Hasan, M. M. (2019). Corporate life cycle research in accounting, finance and corporate governance: A survey, and directions for future research. International Review of Financial Analysis, 69, 188-201. DOI: https://doi.org/10.1016/j.irfa.2018.12.004.

Hasan, M. M., Hossain, M., Cheung, A. W-K, \& Habib, A. (2015). Corporate life cycle and cost of equity capital. Journal of Contemporary Accounting \& Economics, 11(1): 46-60. DOI: https://doi.org/10.1016/j. jcae.2014.12.002.

Koh, S., Durand, R. B., Dai, L., \& Chang, M. (2015). Financial distress: Lifecycle and corporate restructuring. Journal of Corporate Finance, 33, 19-33. DOI: https://doi.org/10.1016/j.jcorpfin.2015.04.004.

Kothari, S. P. (2001). Capital markets research in accounting. Journal of Accounting and Economics, 31(1-3): 105-231. DOI: https://doi.org/10.1016/S0165-4101(01)00030-1.

Lima, A. S., Carvalho, E. V. A., Paulo, E., \& Girão, L. F. A. P. (2015). Estágios do Ciclo de Vida e Qualidade das Informações Contábeis no Brasil. Revista de Administração Contemporânea, 19(3), 398. DOI: http://dx.doi. org/10.1590/1982-7849rac20151711.

Lyle, M. R., Callen, J. L., \& Elliott, R. J. (2013). Dynamic risk, accounting-based valuation and firm fundamentals. Review of Accouning Studies, 18(4): 899-929. DOI: https://doi.org/10.1007/s11142-013-9227-x.

Nekrasov, A., \& Shroff, P. (2009). Fundamentals-based risk measurement in valuation. The Accounting Review, 84(6): 1983-2011. DOI: http://dx.doi.org/10.2139/ssrn.913067.

Ohlson, J. A. (1995). Earnings, book values, and dividends in equity valuation. Contemporary Accounting Research, 11(2): 661-687. DOI: https://doi.org/10.1111/j.1911-3846.1995.tb00461.x.

Saito, R., Villalobos, S., \& Benettui, C. (2008). Qualidade das Projeções dos Analistas Sell side: Evidências Empíricas do Mercado Brasileiro. Revista de Administração da USP, 43(4): 356-369.

Schaberl, P. (2016). Beyond accounting and back: An empirical examination of the relative relevance of earnings and “other" information. Advances in Accounting, 35, 98-113. DOI: https://doi.org/10.1016/j.adiac.2016.08.004.

Vorst, P., \& Yohn, T. (2018). Life cycle models and forecasting profitability and growth. The Accounting Review, 93(6), 357-381. DOI: https://doi.org/10.2308/accr-52091.

\section{Como citar este artigo}

Mikosz, K. da S. C.; Roma, C. M. da S.; Louzada, L. C. \& Macedo, M. R. G. de O. (2019). Previsão de retornos e preços das ações a partir de dados contábeis condicionada ao ciclo de vida das firmas. Revista de Contabilidade e Organizações, 13:e160869. DOI: http://dx.doi.org/10.11606/issn.1982-6486. rco.2019.160869 